\title{
Idade à puberdade e características reprodutivas de novilhas mestiças F1 Holandês x Gir com fenótipos divergentes para consumo alimentar residual
}

\author{
[Age at puberty and reproductive characteristics of F1 Holstein $x$ Gyr crossbred \\ heifers with divergent phenotypes for residual feed intake]
}

\author{
A.P. Fonseca ${ }^{1}$, B.R.C. Alves ${ }^{2}$, M.M. Campos $^{3}$, R.M. Costa ${ }^{4}$, F.S. Machado ${ }^{3}$, \\ L.G.R. Pereira ${ }^{3}$, T.R. Tomich ${ }^{3}$, F.Z. Brandão ${ }^{5}$, Á.M. Borges ${ }^{1}$ \\ ${ }^{1}$ Escola de Veterinária - Universidade Federal de Minas Gerais - Belo Horizonte, MG \\ ${ }^{2}$ University of Nevada - Reno, USA \\ ${ }^{3}$ Embrapa Gado de Leite - Juiz de Fora, MG \\ ${ }^{4}$ Universidade Estadual do Sudoeste da Bahia - Itapetinga, BA \\ ${ }^{5}$ Universidade Federal Fluminense - Rio de Janeiro, RJ
}

\begin{abstract}
RESUMO
A relação da eficiência nutricional com a idade e o peso à puberdade e as características reprodutivas foram estudadas em 35 novilhas F1 Holandês x Gir, em ensaios usando o consumo alimentar residual (CAR) nos períodos pós-desaleitamento (PD) e pós-puberdade (PP). Novilhas PD ( $<<0,0001)$ e $\mathrm{PP}(\mathrm{P}<0,001)$ foram ranqueadas como de alta, intermediária e baixa eficiências e apresentaram CAR de $-0,259-0,014$ e $0,346 \mathrm{~kg} / \mathrm{dia}$ e $-0,848-0,096$ e $0,842 \mathrm{~kg} / \mathrm{dia}$, respectivamente. Novilhas $\mathrm{PD}$ diferiram $(\mathrm{P}<0,05)$ na idade à puberdade entre ranques de alta, intermediária e baixa eficiências, em média, aos 339,7; 346,4; 315,3 dias, respectivamente. Os pesos à puberdade de novilhas PD com alta, intermediária e baixa eficiências foram, em média, de $321 \pm 28,1 \mathrm{~kg}$ e não diferiram $(\mathrm{P}>0,05)$ entre CAR. Não foi verificada correlação entre diâmetro folicular, taxa de crescimento do folículo, número de ondas foliculares no ciclo estral e taxa de gestação à primeira IA com o CARPD ou CARPP. Novilhas F1HG selecionadas para CAR com baixa eficiência podem ter idade à puberdade 10 dias a mais, quando comparadas às novilhas de alta eficiência para CAR. Não foi verificado que novilhas selecionadas para alta ou baixa eficiências alimentares, usando CAR, tivessem algum impacto negativo sobre características reprodutivas avaliadas.
\end{abstract}

Palavras-chave: eficiência alimentar, fertilidade, gado de leite, puberdade, reprodução

\begin{abstract}
The relationship between nutritional efficiency and age and weight at puberty and reproductive traits was studied in $35 \mathrm{Fl}$ Holstein $x$ Gyr heifers, in trials using residual food consumption (CAR) in the post-weaning $(P W)$ and post-pubertal $(P P)$ periods. $P D$ heifers $(P<0.0001)$ and $P P(P<0.001)$ were classified as high, intermediate and low efficiencies and presented CAR of -0.259-0.014 and 0.346kg/day and $-0.848-0.096$ and $0.842 \mathrm{~kg} /$ day, respectively. $P D$ heifers differed $(P<0.05)$ in age at puberty between high, intermediate and low efficiencies, on average, at 339.7, 346.4 and 315.3 days, respectively. The weight at puberty of $P D$ heifers with high, intermediate and low efficiencies were, on average, $321 \pm 28.1 \mathrm{~kg}$ and did not differ $(P>0.05)$ between CAR. No correlation was found between follicular diameter, follicle growth rate, number of follicular waves in the estrous cycle and pregnancy rate at 1st AI with the CARPD or CARPP. F1HG heifers selected for CAR with low efficiency can be aged at puberty 10 days longer when compared to highefficiency heifers for CAR. These results did not show that heifers selected for high or low food efficiency, using CAR, had negative impact on the reproductive characteristics evaluated.
\end{abstract}

Keywords: feed efficiency, milking cattle, puberty, reproduction, fertility

Recebido em 19 de maio de 2019

Aceito em 1 de outubro de 2019

E-mail: adolfoperezfonseca.mvz@gmail.com 


\section{INTRODUÇÃO}

A primeira linhagem do cruzamento entre as raças Holandês e Gir (F1HG) apresenta a máxima heterose, e a utilização desses animais é vista como uma das possíveis estratégias para se aumentar a produtividade leiteira nos países tropicais. Tal premissa baseia-se no fato de os animais F1HG serem rústicos, produtivos, resistentes, longevos, de alta fertilidade $\mathrm{e}$ adaptados ao ambiente tropical (Ruas et al., 2008, 2015). Apesar dos fatores favoráveis às características produtivas, tem-se verificado que animais mestiços Holandês $\mathrm{x}$ Gir atingem a puberdade mais tardiamente em comparação à raça Holandês. Puberdade tardia implica atraso da idade ao primeiro parto, ou seja, idade em que a fêmea passa a ser produtiva dentro de um rebanho bovino. Dados obtidos de 22.380 fêmeas Girolando, participantes de teste de progênie, apontam a idade ao primeiro parto de $35,0 \pm 7,5$ meses e, mais especificamente para as F1HG, de 33,5 \pm 8 meses (Silva et al., 2017), contrastando com a idade de 24 meses, considerada ideal para o primeiro parto de novilhas Holandês.

A alimentação influi diretamente nas características reprodutivas de bovinos (Arthur et al., 2005; Crowley et al., 2011; Shaffer et al., 2011; Hafla et al., 2013). O alto ganho de peso no período de recria pode levar à maturação mais precoce do sistema neuroendócrino reprodutivo (Alves et al., 2015) e ao adiantamento da idade à puberdade (Cardoso et al., 2014). A conversão alimentar, por sua vez, é uma das características consideradas ferramentas de seleção de animais, sendo alternativa para se melhorarem os índices produtivos das fazendas. A maioria dos estudos usando o CAR foram feitos em raças taurinas de corte que fazem avaliações de composição corporal, e tem-se observado que a diferença na gordura corporal e a taxa de metabolismo associada com o CAR podem atrasar a maturidade sexual das novilhas (Shaffer et al., 2011). Portanto, questiona-se a relação entre eficiência alimentar, idade à puberdade e outras características produtivas em novilhas leiteiras, uma vez que a seleção a favor de maior eficiência alimentar poderia implicar manutenção de animais mais tardios ou menos férteis no rebanho.
Também são limitadas as informações relacionadas ao potencial de novilhas F1HG, quando submetidas a manejo nutricional para alto ganho de peso, de atingirem puberdade e peso limiar para a cobertura em idades semelhantes à de animais taurinos.

Os objetivos do presente trabalho foram conhecer o potencial de novilhas mestiças F1HG em alcançarem a puberdade quando submetidas a um regime nutricional intensificado, bem como estudar a relação da eficiência nutricional com a idade à puberdade e com as características reprodutivas de novilhas F1HG.

\section{MATERIAL E MÉTODOS}

Todos os procedimentos envolvendo animais foram aprovados pela Comissão de Ética no Uso de Animais (Ceua, protocolo n ${ }^{\circ} 3403210316$ ), e o experimento foi conduzido na Embrapa Gado de Leite, utilizando-se 35 novilhas F1HG (50\% Holandês e $50 \%$ Gir). Os pesos vivos médios das bezerras ao nascimento e ao desaleitamento, ocorrido aos 90 dias de idade, foram, em média, de $32,7 \pm 6,4 \mathrm{~kg}$ e $101,6 \pm 12,1 \mathrm{~kg}$, respectivamente. Após o desaleitamento, as bezerras foram mantidas em piquete de capim Cynodon plectostachyus, com área de 0,2ha/animal, com fornecimento de água e alimentação em cocho à vontade.

A dieta total (Tab. 1) pós-desaleitamento continha $175,0 \mathrm{~g} \mathrm{~PB} / \mathrm{kg}$ MS e foi composta por $75 \%$ de silagem de milho e $25 \%$ de concentrado à base de soja e sal mineral. $\mathrm{O}$ consumo alimentar individual foi mensurado diariamente de forma automatizada, durante todo o período experimental, utilizando-se sistema de monitoramento Intergado $^{\circledR}$, validado anteriormente por Oliveira et al. (2018). As sobras foram mantidas em $15 \%$ da quantidade fornecida para garantir o consumo ad libitum da dieta. Os animais foram pesados diariamente em balança acoplada ao bebedouro, também de forma automatizada. As dietas foram formuladas segundo recomendações do NRC (Nutrient..., 2001) para ganhos médios de peso vivo de 900g/dia. 
Tabela 1. Composição química do concentrado, silagem de milho e dieta total

\begin{tabular}{llll}
\hline Componentes & Concentrado & $\begin{array}{l}\text { Silagem } \\
\text { de milho }\end{array}$ & $\begin{array}{l}\text { Dieta } \\
\text { total }\end{array}$ \\
\hline $\mathrm{MS}, \mathrm{g} / \mathrm{kg}^{1}$ & 918,6 & 277,9 & 438,1 \\
$\mathrm{~PB}, \mathrm{~g} / \mathrm{kg}^{1}$ & 459,5 & 80,2 & 175,0 \\
$\mathrm{EE}, \mathrm{g} / \mathrm{kg}^{1}$ & 21,2 & 25,1 & 24,1 \\
$\mathrm{FDN}, \mathrm{g} / \mathrm{kg}^{1}$ & 147,5 & 513,2 & 421,7 \\
$\mathrm{FDA}, \mathrm{g} / \mathrm{kg}^{1}$ & 76,3 & 291,6 & 237,8 \\
$\mathrm{~EB}, \mathrm{MJ} / \mathrm{kg}^{1}$ & 18,4 & 16,7 & 17,1 \\
\hline
\end{tabular}

${ }^{1}$ Matéria seca (MS); $\mathrm{PB}=$ proteína bruta; $\mathrm{EE}=$ extrato etéreo; FDN = fibra em detergente neutro; FDA = fibra em detergente ácido; $\mathrm{EB}=$ energia bruta.

Os ensaios de eficiência alimentar foram realizados em dois períodos diferentes, no período pós-desaleitamento (PD), quando a idade média das novilhas era de 149,1 $\pm 30,49$ a 337,6 $\pm 30,2$ dias (Martins, dados não publicados), e no período pós-puberdade $(\mathrm{PP})$, referente às idades de $530,7 \pm 27,8$ a $610,8 \pm 27,8$ dias (Costa, dados não publicados). Os ensaios PD e PP tiveram a duração de 113 dias e 90 dias, respectivamente, e, para cada ensaio, os animais foram ranqueados segundo o parâmetro consumo alimentar residual (CAR). O CAR foi calculado com base na metodologia proposta por Koch et al. (1963), com a diferença entre os consumos de matéria seca observada (CMSobs) e esperada (CMSesp), em que o CAR = CMSobs - CMSesp. O consumo esperado foi obtido por regressão linear múltipla, com base no consumo observado, em função do peso metabólico médio $\left(\mathrm{PV}^{0,75}\right)$ e do ganho de peso vivo médio diário (GPD), em que o intercepto $\beta 0$ e os coeficentes de regressão $\beta 1$ e $\beta 2$ são as médias do grupo, e o resíduo $(\mathcal{E})$ o erro de cada observação, de acordo com a fórmula a seguir:

$$
\text { CMSesp } \left.=\beta 0+\beta 1 \times\left(\mathrm{PV}^{0,75}\right)+\beta 2 \times \text { GPD }\right)+\varepsilon .
$$

Para a avaliação das associações dos parâmetros avaliados e dos índices de eficiência alimentar (EA), os animais foram divididos em três classes: alta, intermediária e baixa eficiências alimentares. A classe CAR de alta eficiência foi composta por animais com CAR $<-0,5$, desvio-padrão abaixo da média; CAR de baixa eficiência foi composta por animais com CAR > 0,5, desvio-padrão acima da média; e, na classe intermediária, os valores de CAR estiveram entre $-0,5$ e $+0,5$, desvio-padrão da média.
A eficiência alimentar do ensaio pósdesaleitamento foi analisada para os parâmetros de idade e peso vivo à puberdade, considerandose os grupos de alta $(n=13)$, intermediária $(n=12)$ e baixa eficiências $(n=10)$. Para o ensaio póspuberdade, buscou-se avaliar sua correlação com as características do diâmetro máximo do folículo pré-ovulatório, da taxa de crescimento folicular, do número de ondas foliculares no ciclo estral e da fertilidade à primeira inseminação artificial, considerando-se novo ranqueamento dos animais, os quais formavam novas classes de alta $(n=10)$, intermediária $(n=17)$ e baixa eficiências alimentares $(n=8)$.

Durante o período peripúbere, a cada sete dias, os animais foram pesados em balança acoplada ao tronco de contenção, e amostras de sangue foram coletadas em tubos contendo EDTA. Após a coleta, o sangue foi centrifugado $(1500 \mathrm{~g} ; 15$ minutos), e o plasma separado e conservado a $20^{\circ} \mathrm{C}$. A partir do peso vivo médio de $266,8 \pm 13,9 \mathrm{~kg}$ (mínimo de $244 \mathrm{~kg}$ e máximo de $303 \mathrm{~kg}$ ), os animais foram submetidos à avaliação ginecológica a cada 14 dias, por dois técnicos treinados, com a utilização de ultrassonografia transretal (Áquila modelo-410853; transdutor linear de 7,5MHz). Ao exame ginecológico, foram avaliados a presença ou ausência de corpo lúteo (CL), o diâmetro do maior folículo ovariano (mm) e o diâmetro médio dos cornos uterinos $(\mathrm{mm})$, mensurados cranialmente, adjacente à bifurcação deles.

A dosagem de progesterona plasmática foi realizada com base nas datas da detecção do corpo lúteo, por meio de radioimunoensaio, utilizandose kit comercial (ImmuChem Coated Tube, ICN Pharmaceuticals, Inc., CA, EUA), seguindo as instruções do fabricante. $\mathrm{O}$ coeficiente de variação intraensaio foi igual a 1 . As novilhas foram consideradas púberes na data da primeira elevação da progesterona acima de $1 \mathrm{ng} / \mathrm{mL}$ (Day et al., 1984), desde que, na semana subsequente, houvesse corpo lúteo confirmado pelo exame ultrassonográfico, ou quando, novamente, a concentração de progesterona estivesse acima de $1 \mathrm{ng} / \mathrm{mL}$. Todos os animais foram avaliados por meio de ultrassonografia por pelo menos 28 dias antes da data da puberdade. Seis animais foram excluídos da análise de puberdade em decorrência de problema de saúde $(n=1)$, perda de amostra ou problema durante a dosagem de progesterona $(n=3)$, ou por finalização do período experimental 
antes de atingirem a puberdade $(\mathrm{n}=2)$. Uma novilha apresentou concentração de progesterona acima de $1 \mathrm{ng} / \mathrm{mL}$ na data da primeira dosagem hormonal e, portanto, foi considerada púbere na data da referida dosagem. Dessa forma, o número de animais utilizados para as análises referentes à idade à puberdade foi de 29 novilhas.

As avaliações reprodutivas da dinâmica folicular ovariana no período pós-púbere foram realizadas nas 35 novilhas quando essas tinham, em média, $17,69 \pm 0,93$ meses de idade, $415,06 \pm 34,84 \mathrm{~kg}$ de peso vivo (PV) e escore corporal (ECC) 3,76 $\pm 0,3$. A ultrassonografia transretal foi realizada a partir do dia do estro natural (Mindray, modelo DP2200; transdutor linear de 5.0MHz). Nesse período, a manifestação do estro foi monitorada visualmente três vezes ao dia, durante 30 minutos: manhã $(7 \mathrm{~h}-7 \mathrm{~h} 30)$, meio-dia $(12 \mathrm{~h}-12 \mathrm{~h} 30)$ e à tarde (17h - 17h30). A partir da detecção do estro, as novilhas foram submetidas à ultrassonografia a cada seis horas, para determinação do intervalo estro-ovulação, que foi determinado como o período médio (horas) da última detecção do folículo e seu desaparecimento do ovário. $\mathrm{O}$ intervalo ovulatório foi definido como o número de dias entre duas ovulações consecutivas, seguidas pela formação do CL (Rhodes et al., 1995).

No estudo da dinâmica folicular, as novilhas foram submetidas à ultrassonografia diária durante um ciclo estral completo, a partir do dia do estro (dia 0). Foram registrados diariamente os diâmetros $(\mathrm{mm})$ máximos do maior e do segundo maior folículo de cada ovário, com o objetivo de se estudar o número de ondas foliculares por ciclo estral e a taxa de crescimento folicular ( $\mathrm{mm} / \mathrm{dia})$, segundo Ginther et al. (1989) e Borges et al. (2001). Nos dias 0 (estro) e 10 (diestro) do ciclo estral, foram avaliados os diâmetros médios dos cornos uterinos, usando-se a mesma metodologia do período peripúbere. Após o estudo da dinâmica folicular ovariana durante o ciclo estral natural, no $10^{\circ}$ dia após a ovulação, os animais receberam injeção i.m. de $150 \mu \mathrm{g}$ de D-cloprostenol (Croniben ${ }^{\circledR}$, Lab. Biogênesis Bagó, Brasil), no intuito de se avaliarem os intervalos da aplicação do agente luteolítico ao estro e à ovulação. Para tal análise, 30 estros induzidos com $\mathrm{PGF}_{2 \alpha}$ foram considerados.

Para as inseminações artificiais, as novilhas foram submetidas a protocolo hormonal, iniciado em dia aleatório do ciclo estral no período pós-púbere, de acordo com o descrito a seguir: dia 0 (D0), 8h, inserção de implante intravaginal de $1 \mathrm{~g}$ de progesterona $\left(\right.$ Sincrogest $^{\circledR}$, Lab. Ouro Fino, Brasil) e aplicação de $2 \mathrm{mg}$ de benzoato estradiol (BE) (Estrogin ${ }^{\circledR}$, Lab. Farmavet, Brasil); D7: duas doses de $150 \mu \mathrm{g}$ de D-cloprostenol (Croniben ${ }^{\circledR}$, Lab. Biogênesis Bagó, Brasil), sendo a primeira às $8 \mathrm{~h}$ e a segunda às $17 \mathrm{~h}$; D8, $8 \mathrm{~h}$ : $1 \mathrm{mg}$ de cipionato de estradiol (ECP) (SincroCP ${ }^{\circledR}, \mathrm{Lab}$ Ouro Fino, Brasil) e retirada do implante intravaginal. No dia da retirada do implante, foi avaliado o diâmetro do maior folículo (préovulatório) e, a partir desse momento, o estro foi monitorado continuamente até a detecção do último animal em estro. As inseminações artificiais foram realizadas 12 horas após a detecção da primeira aceitação de monta, com o sêmen de um único touro da raça Holandês, de fertilidade comprovada. O diagnóstico de gestação foi realizado por ultrassonografia aos 28 dias após a inseminação artificial e reconfirmado aos 60 dias de gestação.

As análises das relações do consumo alimentar residual (CAR) e dos parâmetros reprodutivos foram realizadas utilizando-se o software SAS (versão 9.4, SAS Institute Inc., Cary, NC). Médias, desvios-padrão (DP), normalidade e homogeneidade de variância foram avaliados para todas as variáveis, utilizando-se o procedimento Univariate. Os coeficientes de correlação de Pearson entre idade à puberdade, peso à puberdade, tamanho máximo do folículo préovulatório, taxa de crescimento folicular e CAR foram calculados usando-se o procedimento CORR. Médias foram testadas usando-se o teste de Tukey-Krumer. Variáveis não paramétricas (taxa de gestação à primeira IA) e análises paramétricas (número de ondas foliculares) foram avaliadas pelo teste de Kruskal-Wallis (comparação entre grupos), considerando-se $\alpha=0,05$. Coeficientes de correlação de Spearman para taxa de gestação à primeira IA e número de ondas foliculares e CAR também foram calculados. As análises estatísticas dos parâmetros reprodutivos foram realizadas utilizando-se o software SAEG, versão 9.1 (Sistema..., 2007). Dados de diâmetro de cornos uterinos e dos folículos ovarianos no período prépúbere foram agrupados em três idades $(-49 \mathrm{a}-42$, -35 a -28 e -21 a -14 dias da puberdade) e, juntamente com os dados obtidos nos dias 0 e 10 do período pós-púbere, foram comparados 
levando-se em consideração o período de mensuração como variável independente, por ANOVA, utilizando-se o teste Student-NewmanKeuls (SNK) para se testarem as diferenças de médias. Análises de correlação de Pearson foram realizadas entre os diâmetros dos cornos uterinos e dos maiores folículos ovarianos detectados no mesmo momento da análise. As médias de intervalos da detecção do estro à ovulação e intervalos ovulatórios foram comparadas em função tipo de estro (natural $\mathrm{x}$ induzido com luteolítico), pelo teste F.

\section{RESULTADOS E DISCUSSÃO}

No pós-desaleitamento, novilhas de alta, intermediária e baixa eficiências (dados em $\mathrm{kg} / \mathrm{MS} /$ dia) apresentaram consumo alimentar residual (CAR) de -0,259, -0,014 e 0,346, respectivamente, com diferenças $(\mathrm{P}<0,0001)$ entre os grupos. Da mesma forma, na póspuberdade, houve diferença $(\mathrm{P}<0,0001)$ para $\mathrm{O}$ consumo alimentar residual, considerando-se os grupos de novilhas de alta $(-0,848)$, intermediária $(-0,096)$ e baixa $(0,842)$ eficiências (valores em $\mathrm{kg} / \mathrm{MS} /$ dia). As idades à puberdade diferiram $(\mathrm{P}<0,0057)$ entre novilhas $\mathrm{F} 1 \mathrm{HG}$ ranqueadas como de alta, intermediária e baixa eficiências alimentares no período pós-desaleitamento. A puberdade ocorreu, em média, aos 339,8, 346,4 e 329,6 dias, respectivamente, para os três grupos supracitados (Tab. 2).

Tabela 2. Idades e pesos vivos (médias \pm desvios-padrão) à puberdade de novilhas leiteiras F1HG com fenótipo divergente para consumo alimentar residual (CAR) no período pós-desaleitamento (PD)

\begin{tabular}{llllll}
\hline \multirow{2}{*}{$\begin{array}{l}\text { Parâmetros de eficiência produtiva } \\
\text { e reprodutiva }\end{array}$} & $\begin{array}{l}\text { Grupos de CAR no período pós-desaleitamento } \\
\text { Eficiência } \\
\text { alta }\end{array}$ & $\begin{array}{l}\text { Eficiência } \\
\text { intermediária }\end{array}$ & $\begin{array}{l}\text { Eficiência } \\
\text { baixa }\end{array}$ & EPM & P-valor \\
\hline Consumo alimentar residual $(\mathrm{kg} / \mathrm{d})$ & $-0,259 \mathrm{c}$ & $-0,014 \mathrm{~b}$ & $0,346 \mathrm{a}$ & 0,045 & $<0,0001$ \\
Idade à puberdade (dias) & $339,78 \pm 32,1 \mathrm{~b}$ & $346,43 \pm 31,6 \mathrm{a}$ & $329,60 \pm 34,1 \mathrm{c}$ & 5,929 & 0,005 \\
Peso vivo à puberdade $(\mathrm{kg})$ & $332 \pm 31,0 \mathrm{a}$ & $317 \pm 21,2 \mathrm{a}$ & $315,3 \pm 29,5 \mathrm{a}$ & 5,522 & 0,11 \\
\hline
\end{tabular}

a,b,c Valores seguidos por letras minúsculas distintas na coluna diferem $(\mathrm{P}<0,05)$ pelo teste de Tukey-Krumer.

Os resultados indicam que novilhas F1HG selecionadas para CAR com alta eficiência podem ter idade à puberdade 10 dias mais tarde em relação às novilhas de baixa eficiência para CAR. Novilhas taurinas de corte com baixa eficiência para CAR alcançaram a puberdade mais precocemente do que as de alta eficiência para CAR (414 $\pm 3,38 v s .427 \pm 4,67$ dias) (Shaffer et al., 2011), o que corrobora os dados do presente experimento. No experimento de Shaffer et al. (2011), verificou-se que cada aumento de uma unidade no CAR correspondeu à diminuição de 7,5 dias na idade à puberdade. Crowley et al. (2011), ao estudarem desempenhos de 94.936 vacas mestiças taurinas de corte, encontraram correlação genética negativa entre idade ao primeiro parto, taxa de conversão alimentar e CAR. Foi sugerido que a seleção para melhorar a eficiência alimentar pode atrasar a puberdade, aumentando a idade ao primeiro parto. No sistema estudado no presente experimento, o atraso de 10 dias na idade à puberdade implica aumento de dias não produtivos, o que poderia, em hipótese a ser investigada futuramente, ser compensado pelo menor gasto com alimentação desses animais mais eficientes na conversão.

No presente estudo, a idade à puberdade das novilhas F1HG foi, em média, de 340,2 232 dias (11,3 $\pm 1,0$ meses), sendo o mínimo de 272 e o máximo de 396 dias. Novilhas da raça Holandesa, quando submetidas a regime nutricional para alto ganho de peso, têm a capacidade de atingir a puberdade entre nove e 12 meses (Chelikani et al., 2003). No presente estudo, a idade à puberdade foi semelhante à de fêmeas da raça Holandesa, o que comprova o potencial de animais leiteiros de $50 \%$ de genética zebuína apresentarem fenótipos relacionados à maturidade sexual semelhante a animais $100 \%$ taurinos.

Os pesos vivos à puberdade não diferiram $(\mathrm{P}>0,05)$ entre novilhas ranqueadas no pósdesaleitamento como de alta, intermediária e baixa eficiências, sendo, em média, de $321 \pm 28,1 \mathrm{~kg}$ (Tab. 3). Os ganhos de peso vivo médio diário, do pós-desaleitamento até a puberdade, foram de $0,83 \pm 0,11 ; 0,84 \pm 0,12$ e $0,85 \pm 0,07 \mathrm{~kg} / \mathrm{dia}$ para novilhas de alta, 
intermediária e baixa eficiências, respectivamente, sendo considerados satisfatórios para todos os grupos. Basarab et al. (2007) verificaram que vacas mais eficientes (CAR negativo) consumiram menos alimento, passavam menos tempo se alimentando e tiveram crescimento e peso vivo semelhantes às menos eficientes (CAR positivo)

Tabela 3. Parâmetros ponderais do nascimento à puberdade de novilhas F1HG

\begin{tabular}{lllllll}
\hline \multicolumn{7}{c}{ Parâmetros } \\
\hline $\begin{array}{l}\mathrm{PN}(\mathrm{n}=35) \\
(\mathrm{kg})\end{array}$ & $\begin{array}{l}\mathrm{PD}(\mathrm{n}=34) \\
(\mathrm{kg})\end{array}$ & $\begin{array}{l}\text { ID }(\mathrm{n}=34) \\
(\text { dias })\end{array}$ & $\begin{array}{l}\text { GPD }(\mathrm{n}=34) \\
(\mathrm{kg})\end{array}$ & $\begin{array}{l}\text { GPP }(\mathrm{n}=29) \\
(\mathrm{kg})\end{array}$ & $\begin{array}{l}\text { IP }(\mathrm{n}=29) \\
(\text { dias })\end{array}$ & $\begin{array}{l}\text { PP } \\
(\mathrm{n}=29)\end{array}$ \\
$32,7 \pm 6,4$ & $101,6 \pm 6,4$ & $81 \pm 2,6$ & $0,85 \pm 0,2$ & $0,87 \pm 0,1 \mathrm{a}$ & $340,2 \pm 32$ & $321 \pm 28$ \\
\hline
\end{tabular}

$\mathrm{PN}=$ Peso ao nascimento; $\mathrm{PD}=$ peso ao desaleitamento; $\mathrm{ID}=$ idade ao desaleitamento; $\mathrm{GPD}=$ ganho de peso vivo médio diário do nascimento ao desaleitamento; $\mathrm{GPP}$ = ganho de peso vivo médio diário do desaleitamento à puberdade; $\mathrm{IP}=$ idade à puberdade; $\mathrm{PP}=$ peso à puberdade .

$\mathrm{O}$ peso vivo médio à puberdade observado no presente estudo equivaleu a $61,7 \%$ do peso adulto (Ruas et al., 2007) de uma fêmea F1HG. Teodoro et al. (1984) verificaram, em animais F1HG, idade e peso vivo à puberdade de 725 dias e $334 \mathrm{~kg}$, respectivamente. Os dados do presente estudo corroboram a ideia de que, em animais mestiços Holandês- Gir, o peso vivo, e não a idade, é fator mais importante na determinação da idade à puberdade, como já descrito para a raça Holandesa (Chelikani et al., 2003) ou para as raças zebuínas (Galina e Arthur, 1989).

O ganho de peso vivo da desmama à puberdade foi, em média, de $0,87 \pm 0,1 \mathrm{~kg}$ (mínimo de $0,65 \mathrm{~kg}$ e máximo de $1,0 \mathrm{~kg}$ ). Novilhas submetidas a baixo ganho médio diário de peso vivo $(0,350 \mathrm{~kg})$ atingem a puberdade próximo de 19 meses de idade, quando comparadas com novilhas ganhando peso igual ou superior a $1 \mathrm{~kg} / \mathrm{dia}$, ou seja, até os nove meses de idade (Chelikani et al., 2003). O aumento no ganho de peso vivo durante o período pré-púbere leva à maturação precoce do sistema neuroendócrino e à puberdade (Alves et al., 2015; Cardoso et al., 2015), o que antecipa a liberação de LH em alta frequência (Cardoso et al., 2015). O presente estudo mostrou que novilhas F1HG apresentaram resposta favorável à nutrição, atingindo idade à puberdade, em média, aos 11,3 $\pm 1,0$ meses. Os parâmetros foliculares e uterinos de novilhas mestiças F1HG em diferentes momentos, na pré e pós-puberdade, estão contidos na Tab.4.

Tabela 4. Diâmetros (mm; médias \pm desvios-padrão) do maior folículo e dos cornos uterinos em novilhas F1 HG na pré e pós-puberdade

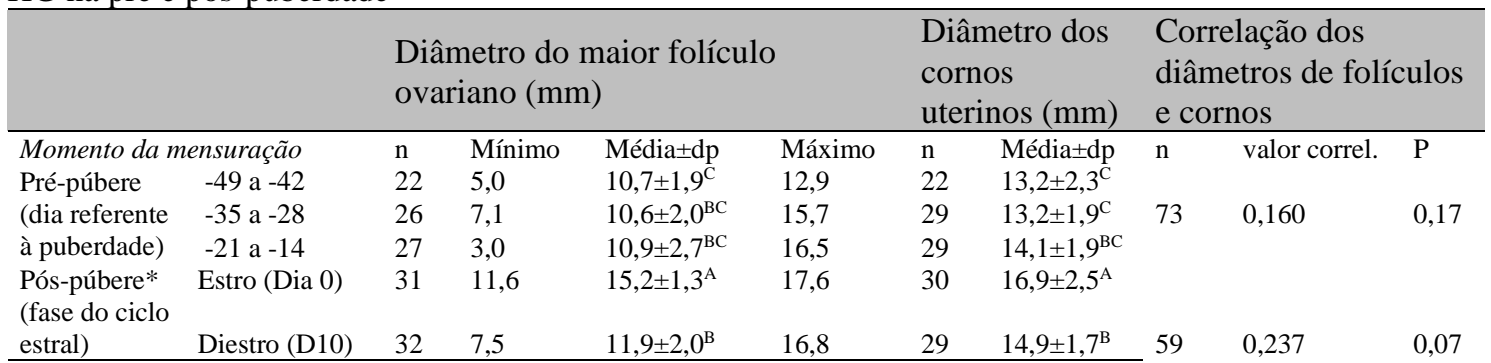

*Período mínimo de quatro meses após a puberdade. Médias seguidas por letras distintas na coluna diferem $(\mathrm{P}<0,05)$ entre si pelo teste de Tukey.

Aproximadamente 50 dias antes da puberdade, é possível se verificarem folículos com diâmetro compatível com o período pré-ovulatório. Em novilhas púberes mestiças Holandês-Zebu, Borges et al. (2001) reportaram tamanhos médios de folículos dominantes de 13,3 $\pm 1,4$ e
$11,8 \pm 1,3 \mathrm{~mm}$ para fêmeas com ciclos de duas ondas e três ondas, respectivamente.

O diâmetro dos cornos uterinos dos 49 aos 28 dias anteriores à puberdade foi semelhante, porém inferior aos do período pós-púbere. Verificou-se 
aumento do diâmetro dos cornos a partir dos 21 dias anteriores à puberdade, o que é esperado durante o desenvolvimento peripuberal do sistema genital de bovinos (Honaramooz et al., 2004). No período pós-púbere, foi verificada diferença $(\mathrm{P}<0,05)$ no diâmetro dos cornos uterinos em função da fase do ciclo estral, caracterizado pelo aumento de $15 \%$ durante o estro, em relação ao diâmetro no diestro. A maturação uterina depende do estímulo alternado da progesterona e do estrógeno, que acontece após a primeira ovulação seguida de corpo lúteo funcional (Spencer e Bazer, 2004).

Um experimento com novilhas Hereford mostrou que o padrão de crescimento uterino tem alta correlação com o tamanho do maior folículo ovariano, sugerindo que o aumento de estradiol, a partir dos folículos maiores, estimula o crescimento e o desenvolvimento do útero
(Honaramooz et al., 2004). No presente estudo, essa correlação não foi observada no período prépuberal, mas, sim, no período pós-púbere, que correspondeu a, no mínimo, quatro meses após a puberdade, quando se espera que as novilhas já tenham atingido a maturidade sexual. Necessitase, portanto, de um estudo mais aprofundado entre o tamanho folicular de novilhas mestiças durante a pré-puberdade e sua relação com as concentrações circulantes de estradiol e com o estímulo da maturação uterina.

No período pós-púbere, o diâmetro e a taxa de crescimento do folículo pré-ovulatório e a taxa de gestação à primeira inseminação não diferiram $(\mathrm{P}>0,05)$ para novilhas com CAR para eficiências alta, intermediária e baixa, sendo, em média, de $15,07 \pm 1,28 \mathrm{~mm}, \quad 1,40 \pm 0,39 \mathrm{~mm} / \mathrm{dia}$ e $80 \%$, respectivamente (Tab. 5).

Tabela 5. Parâmetros foliculares (médias \pm desvios-padrão) e taxa de gestação de novilhas mestiças F1HG com fenótipo divergente para consumo alimentar residual (CAR) no período pós-puberdade

\begin{tabular}{llllll}
\hline $\begin{array}{l}\text { Parâmetros de eficiência produtiva } \\
\text { reprodutiva }\end{array}$ & $\begin{array}{l}\mathrm{e} \text { Grupos de CAR período pós-puberdade } \\
\text { Eficiência } \\
\text { alta }\end{array}$ & $\begin{array}{l}\text { Eficiência } \\
\text { intermediária }\end{array}$ & $\begin{array}{l}\text { Eficiência } \\
\text { baixa }\end{array}$ & EPM & P-valor \\
\hline Consumo alimentar residual (kg/d) & $-0,848 \mathrm{c}$ & $-0,096 \mathrm{~b}$ & $0,842 \mathrm{a}$ & 0,115 & $<0,0001$ \\
Diâmetro do folículo pré-ovulatório (mm) & $14,74 \pm 1,6$ & $14,89 \pm 1,0$ & $15,60 \pm 1,3$ & 0,224 & 0,30 \\
Taxa crescimento do folículo (mm/dia) & $1,39 \pm 0,4$ & $1,38 \pm 0,4$ & $1,44 \pm 0,3$ & 0,068 & 0,93 \\
Taxa de gestação à 1- IA $(\%)$ & $75,0 \pm 4,0$ & $88,0 \pm 3,0$ & $70,0 \pm 4,0$ & 0,068 & 0,49 \\
\hline
\end{tabular}

$\overline{a, b, c}$ Valores seguidos por letras minúsculas distintas na linha diferem $(\mathrm{P}<0,05)$ pelo teste de Tukey-Krumer.

Estudos anteriores mostraram que novilhas de alta e baixa eficiências alimentares, categorizadas de acordo com o CAR, não apresentaram diferenças nas taxas de detecção de estro, concepção e gestação (Shaffer et al., 2011); da mesma forma, vacas de alto e baixo CAR apresentaram taxas de gestação semelhantes (Arthur et al., 2005). Em um estudo retrospectivo, que analisou 94.936 vacas mestiças taurinas, não se verificou correlação entre seleção de vacas para eficiência alimentar e fertilidade, sugerindo que a seleção para melhoria da eficiência alimentar não possui efeito prejudicial sobre o desempenho reprodutivo, exceto para o atraso à puberdade $\mathrm{e}$, consequentemente, ao primeiro parto (Crowley et al., 2011). No presente estudo, também não se observou correlação $(\mathrm{P}>0,05)$ entre idade e peso à puberdade, diâmetro do folículo pré-ovulatório (mm), taxa de crescimento do folículo ( $\mathrm{mm} / \mathrm{dia})$ e taxa de gestação primeira IA de novilhas com CAR pós-desaleitamento e pós-puberdade. Os dados observados no presente estudo são concordantes com os estudos supracitados, indicando que a seleção de novilhas mais eficientes com relação ao CAR não implica redução da fertilidade do rebanho.

No estudo da dinâmica folicular ovariana, 39,4\% e $60,6 \%$ das novilhas apresentaram ciclos estrais com duas e três ondas foliculares, respectivamente. Dados semelhantes foram encontrados por Borges et al. (2001), em novilhas mestiças Holandês-Zebu. As taxas de detecção de estros foram de $94 \%(n=33 / 35)$ e $84 \%(n=30 / 35)$, respectivamente, para estros naturais (observação ininterrupta) ou induzidos com $\mathrm{PGF}_{2 \alpha}$ (observação três vezes ao dia por $30 \mathrm{~min}$ ). Tais dados indicam alta eficiência de detecção de estro e possível ocorrência de cio silencioso nas novilhas F1HG. No presente estudo, após o protocolo associando progesterona e estradiol, as novilhas F1HG manifestaram estro e foram inseminadas, em 
média, 31,6 $6 \pm 5,2$ horas após a retirada do implante de P4 (43,6 $\pm 5,2$ após aplicação de ECP). Os intervalos do estro à ovulação foram de $25,4 \pm 4,13 \mathrm{~h}$ para estros naturais e $25,3 \pm 6,23 \mathrm{~h}$ para estros induzidos com luteolítico $(\mathrm{P}>0,05)$. Os intervalos da aplicação da $\mathrm{PGF}_{2 \alpha}$ ao estro e à ovulação das novilhas F1HG foram de $94,90 \pm 29,87 \mathrm{~h}$ e $120,20 \pm 20 \mathrm{~h}$.

A taxa de concepção aos 28 após IA foi de $80 \%$ (28/35), não havendo morte embrionária até os 60 dias de gestação. Novilhas zebuínas que utilizaram protocolo para inseminação artificial em tempo fixo com P4 e ECP ou BE tiveram porcentagem de gestação total de $40 \%$. A porcentagem para novilhas que usaram protocolo com ECP foi de $51 \%$ de gestação e, para novilhas usando BE, foi de $30 \%$ (Peralta-Torres et al., 2010). Os dados do presente estudo demonstram alto potencial de fertilidade de novilhas F1HG após utilização de protocolo hormonal para indução de estro no período pós-púbere.

\section{CONCLUSÕES}

Novilhas mestiças F1HG apresentam potencial para atingirem a puberdade até os 13 meses de idade, quando são bem alimentadas, além de alta fertilidade após a utilização de protocolo hormonal seguido pela observação de estro e inseminação artificial. A seleção de novilhas de alta eficiência alimentar, utilizando-se o CAR como parâmetro, pode atrasar a idade à puberdade do rebanho, sem outros prejuízos ao desempenho reprodutivo até a primeira gestação.

\section{AGRADECIMENTOS}

À Embrapa/Gado de Leite, à Capes, ao CNPq, à Escola de Veterinária/UFMG e à UFF.

\section{REFERÊNCIAS}

ALVES, B.R.C.; CARDOSO, R.C.; PREZOTTO, L.D. et al. Elevated body weight gain during the juvenile period alters neuropeptide Ygonadotropin-releasing hormone circuitry in prepuberal heifers. Biol. Reprod., v.92, p.1-10, 2015.
ARTHUR, P.F.; HERD, R.M.; WILKINS, J.F. et al. Maternal productivity of Angus cows divergently selected for post-weaning residual feed intake. Aust. J. Exp. Agrar., v.45, p.985-993, 2005.

BASARAB, J.A.; CARTNEY, D.; OKINE, E.K. et al. Relationships between progeny residual feed intake and dam productivity traits. Can. J. Anim. Sci., v.87, p.489-502, 2007.

BORGES, A.M.; TORRES, C.A.A.; RUAS, J.R.M. et al. Dinâmica folicular ovariana novilhas mestiças Holandês-Zebu. Arq. Bras. Med. Vet. Zootec., v.53, p.595-604, 2001.

CARDOSO, R.C.; ALVES, B.R.C.; PREZOTTO, L.D. et al. Use of a stair-step compensatory gain nutritional regimen to program the onset of puberty in beef heifers. J. Anim. Sci., v.92, p.2942-2949, 2014.

CARDOSO, R.C.; ALVES, B.R.C.; SHARPTON, S.M. et al. Nutritional programming of accelerated puberty in heifers: Involvement of pro-opiomelanocortin neurons in the arcuate nucleus. J. Endocrinol., v.27, p.647$657,2015$.

CHELIKANI, P.K.; AMBROSE J.D.; KANNELLY J.J. Effect of dietary energy and protein density on body composition, attainment of puberty, and ovarian follicular dynamics in dairy heifers. Theriogenology, v.60, p.707-725, 2003.

CROWLEY, J.J.; EVANS, R.D.; MC HUGH, N. et al. Genetic relationships between feed efficiency in growing males and beef cow performance. J. Anim. Sci., v.89, p.3372-3381, 2011.

DAY, M.L.; IMAKAWA, K.; GARCIAWINDER, M. et al. Endocrine mechanisms of puberty in heifers: estradiol negative feedback regulation of luteinizing hormone secretion. Biol. Reprod., v.2, p.332-341, 1984.

GALINA, C.S.; ARTHUR, G.H. Review of cattle reproduction in the tropics. Part 1. Puberty and age at first calving. Anim. Breed. Abstr., v.57, p.583-590, 1989.

GINTHER, O.J.; KASTELIC, J.P.; KNOPF, L. Composition and characteristics follicular waves during the bovine estrous cycle. Anim. Reprod. Sci., v.20, p.187-200, 1989. 
HAFLA, A.D.; CARSTENS, G.E.; FORBES, T.D.A. et al. Relationships between postweaning residual feed intake in heifers and forage use, body composition, feeding behavior, physical activity, and heart rate of pregnant beef females. J. Anim. Sci., v.91, p.5353-5365, 2013.

HONARAMOOZ, A.; ARAVINDAKSHAN, J.; CHANDOLIA, R.K. et al. Ultrasonographic evaluation of the pre-pubertal development of the reproductive tract in beef heifers. Anim. Reprod. Sci., v.80, p.15-29, 2004.

KOCH, R.M.L.A.; SWIGER, D.; CHAMBERS, K.E. et al. Efficiency of feed use in beef cattle. $J$. Anim. Sci., v.22, p.486-494, 1963.

NUTRIENT requirements of dairy cattle. 7.ed. Washington: National Academic Press, 2001. 408 p.

OLIVEIRA, B.R.; RIBAS, M.N.; MACHADO, F.S. et al. Validation of a system for monitoring individual feeding and drinking behaviour and intake in young cattle. Animal, v.12, p.634-639, 2018.

PERALTA-TORRES, J.A.; AKÉ-LÓPEZ, J.R.; CENTURIÓN-CASTRO, F.G. et al. Comparación del cipionato de estradiol vs benzoato de estradiol sobre la respuesta a estro y tasa de gestación en protocolos de sincronización con CIDR en novillas y vacas Bos indicus. Univ. Cienc., v.26, p.163-1692010.

RHODES, F.M.; FITZPATRICK, L.A.; ENTWISTLE, K.W. et al. Sequential changes in ovarian follicular dynamics in Bos indicus heifers before and after nutritional anoestrus. J. Reprod. Fertil., v.104, p.41-49, 1995.
RUAS, J.R.M.; CARVALHO, B.C.; SILVA FILHO, J.M. et al. Efeito da base genética materna e da estação de parição sobre variáveis produtivas de fêmeas primíparas HolandêsxZebu. Arq. Bras. Med. Vet. Zootec., v.59, p.218-224, 2007.

RUAS, J.R.M.; SILVA, E.A.; QUEIROZ, D.S. et al. Considerações sobre a produção de leite com vacas F1 Holandês x Gir. Inf. Agropecu., v.36, p.51-58, 2015.

RUAS, J.R.M.; SILVA, M.A.; FERREIRA, J.J. et al. Desempenho produtivo e reprodutivo de vacas F1 Holandês x Zebu em rebanhos da EPAMIG. ENCONTRO DE PRODUTORES DE GADO LEITEIRO F1. 6., Belo Horizonte. Anais... Belo Horizonte: PUC Minas, 2008. p.146-182.

SHAFFER, K.S.; TURK, P.; WAGNER, W.R. et $a l$. Residual feed intake, body composition, and fertility in yearling beef heifers. J. Anim. Sci., v.89, p.1028-1034, 2011.

SILVA, M.V.G.B.; MARTINS, M.F.; CEMBRANELLI, M.A.R. et al. Programa de melhoramento genético da raça F1 HG. Avaliação genética de vacas, 2017. Juiz de Fora: Embrapa Gado de Leite, 2017. 42p.

SISTEMA para análises estatísticas-SAEG. Versão 9.1. Viçosa: UFV / Fundação Arthur Bernardes, 2007. 142p.

SPENCER, T.E.; BAZER, F.W. Uterine and placental factors regulating conceptus growth in domestic animals. J. Anim. Sci., v.82, p.E4-E13, 2004.

TEODORO, R.L.; LEMOS, A.M.; BARBOSA, E.T. et al. Comparative performance of six Holstein Friesian x Guzera grades in Brazil: 2 traits related the onset the sexual function. Anim. Prod., v.38, p.165-170, 1984. 